\title{
ON A MODIFICATION OF THE HILGER SECTOR PHOTOM- ETER METHOD FOR MEASURING ULTRA-VIOLET ABSORPTION AND ITS APPLICATION IN THE CASE OF CERTAIN DERIVATIVES OF FLUORAN.
}

By H. E. Howe.

COLOR in chemical compounds, or the lack of it, has been made the basis for decisions regarding the molecular structure of the compounds. Since color depends upon the absorption of light in the visible part of the spectrum only, it would seem that ultimate conclusions as to the relation between structure and absorption will necessitate a knowledge of the complete absorption spectrum from ultra-violet to infra-red.

The work described was begun for the purpose of studying the effect on the ultra-violet absorption of a group of related phthaleins when halogen or hydroxyl groups are substituted for hydrogen in the molecule and of determining what change in the absorption spectrum occurs when colorless alcoholic solutions of these phthaleins become colored upon the addition of alkali. The absorption curves presented in this paper are too few to form a basis for general conclusions, but they serve to illustrate the method of work.

In connection with the work of determining the ultra-violet absorption of the solutions studied, two physical problems were met. These were (I) the establishment of a method by which measurements can be made with the Hilger sector photometer and (2) the development of a source of light satisfactory for use in the ultra-violet. The author has found that the photographic plate, the use of which in photometric measurements is usually considered questionable, integrates intermittent exposures in such a way that the comparison of two intensities can be made directly in terms of sector openings provided that the time from the beginning to the end of the exposures is the same and that the two exposures produce equal blackenings of the plate. The aluminum spark under water, described by Henri, ${ }^{1}$ has been improved until it has become a very strong source, easy of control and reasonably constant. The spectrum which this source gives is continuous with only a few lines superposed and can be used, with long exposures, to .2I $\mu$.

${ }^{1}$ V. Henri, Phys. Zeit., I4, p. 5 I6, I913. 
The Manipulative Problem.

In plotting absorption curves a method proposed by Baly and Detsch ${ }^{1}$ has been followed by chemists almost without exception. Photographs of the spectrum of the light source and of the transmission spectra of layers of different thickness are taken on the same negative, the same time of exposure being used throughout. The "limits of absorption," $i . e$. , the points where the transmission spectrum has blackened the plate the same as the unmodified spectrum, are marked on each photograph, and these limits are plotted against the relative thicknesses of the most dilute solution used.

The great difficulty of this method lies in the determination of the limits of absorption, because the two parts of the plate being compared are so far apart. This difficulty, which is especially noticeable if the source used gives a line spectrum, is illustrated in Plate I. The upper photograph was made using an iron spark and a solution of anthracene in alcohol, with thicknesses from I $\mathrm{mm}$. to $50 \mathrm{~mm}$. One is inclined to mistake for absorption bands what are really weak places in the spectrum of the source.

The second photograph was made in the same manner as the first, except that the aluminum spark under water was used. The superiority of the second source is apparent. But with either source there is still an objection to the method, for no allowance is made for the absorption of the solvent, and the error is a varying one as the thickness of the

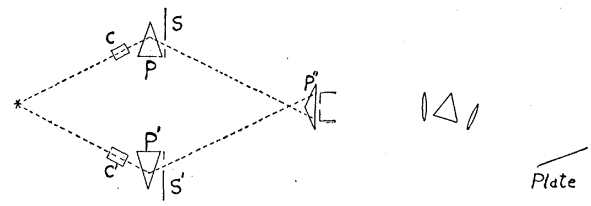

Fig. 1.

absorbing layer changes. With alcohol as a solvent the error in the extreme ultra-violet is considerable. Curves plotted from either of the two negatives here reproduced show a much greater absorption in the short wave-lengths than does the true curve for the solute alone.

The third photograph in Plate $I$. is from a negative made with the apparatus to be described. From such a negative the true absorption of the solute can be found.

The arrangement of the parts of this apparatus is shown in Fig. I, which for convenience is made to show the spectrograph in plan and the photometer attachment in elevation. Two beams from the source pass

${ }^{1}$ Baly and Detsch, Trans. Chem. Soc., 85, p. 1039, I904. 
through the quartz prisms $P$ and $P^{\prime}$, and when incident on the small biprism $P^{\prime \prime}$, are so bent that they are focused by the spectrograph as two narrow spectra with a fine dividing line between. The sector $S^{\prime}$ has a constant opening of $180^{\circ} . \quad S$ is variable and has a scale reading in logarithms of the relative openings of $S^{\prime}$ and $S$. The absorbing solution is put into the cell at $C^{\prime}$ and a similar cell, filled with the solvent, is placed at $C$. Exposures through the two cells are made simultaneously. Sixteen exposures are made on one negative, with sector scale readings from o to I.5.

On the developed negative the points of equal blackening are marked and the corresponding frequencies read by means of a transparent frequency scale. To locate the points of equal blackening the negative is laid on an illuminated milk glass plate and a paper screen with a small hole in it is moved along the blackened strip until the point of match is found. A dot is then made with a fine pen. In this way a sort of curve is "spotted" on the negative. The "spotted" negative is then laid over the frequency scale and the frequency of each point of match read. The scale used was made by photographing to proper size a magnified scale drawn from the calibration curve of the spectrograph. The known frequencies of the aluminum lines showing on the negative enables one to place the scale properly under the negative to be examined.

Attempts to read the points of equal blackening by the use of (I) a micrometer microscope and (2) a projection lantern with a cross-hair moving over the negative showed that the spotting method was quicker, easier on the eyes, and quite as accurate.

Great care must be taken to keep the alignment of the apparatus correct. The light must come from a narrow horizontal source (a spark between metal electrodes was used), and a very small displacement of the line source up or down causes inequality of the two beams reaching the plate. When such a displacement occurs, the fact shows up in a test photograph by one of the blackened strips being darker at one end than is the other strip, while at the other end, the inequality is reversed. Thus the error is least in the middle part of the spectrum (between frequencies 3,000 and 4,000). It is in this part of the spectrum that most of the readings reported were taken. As a precaution, the first and last exposures on each negative were made with both cells $C$ and $C^{\prime}$ removed. If the electrodes had become displaced, the negative was discarded.

Calling $I$ and $I^{\prime}$ the intensities of the beams transmitted by the two cells $C$ and $C^{\prime}$, the ratio $I / I^{\prime}$ represents the ratio of intensities of a beam of light before and after its passage through a layer of the dissolved substance, considered alone. If this layer of absorbing molecules is of 
thickness $d$ and concentration $c$ (gram-molecules per liter), we may write

$$
\frac{I}{I^{\prime}}=\mathrm{IO}^{\beta c d} \quad \text { or } \quad \beta=\frac{\mathrm{I}}{c d} \times \log \frac{I}{I^{\prime}}
$$

$\beta$ is called the "molecular absorption constant" (or sometimes, "molecular extinction coefficient"). This relation assumes that the dilution is such that the relative number of active molecules is independent of the concentration, i. e., that Beer's law is followed.

Suppose a photograph taken with $S$ set at $.3, i$.e.,

$$
\text { L g } \frac{\text { opening } S^{\prime}}{\text { opening } S}=.3 \text {, }
$$

and that the spectra obtained cause equal blackening for some wavelength. If the photographic plate integrates the incident energy properly, we can say

$$
\log \frac{\text { light incident on absorbent }}{\text { light transmitted by absorbent }}=.3=\log \frac{I}{I^{\prime}} \text {. }
$$

Reasons for assuming this relation to hold will be discussed in the following paragraphs.

\section{The Photographic Problem.}

In photometry by photographic means one may use the Schwarzschild ${ }^{1}$ relation, $I_{1} t_{1}{ }^{\delta}=I_{2} t_{2}{ }^{\delta}$, when the two regions of the plate to which the $I$ 's and $t$ 's apply are blackened equally by continuous exposures. The exponent $\delta$ depends upon the brand of plate. Its possible variation with the handling of the plate during exposure and development, and the possible growth and decay of the latent image after exposure make the application of the relation somewhat questionable.

The only relation that will unquestionably apply was stated by Hartmann somewhat as follows: two beams of light which produce equal blackening of two areas of the plate may be taken as equal provided (I) the two beams are of the same wave-length; (2) they act upon closely adjacent parts of the plate; (3) they are of constant intensity, or vary together; (4) they act simultaneously. These conditions are imposed because (I) the sensibility of the plate varies with the quality of the light, (2) the plate may vary from point to point, (3) and (4) the relation between $I, t$, and the blackening is uncertain.

All of these conditions except the fourth are met by the Hilger apparatus. The total time from the beginning to the end of exposure is the same for the two beams. But the beams are of unequal intensities, and the actual times during which they act on the plate are unequal,

1 Schwarzschild, Astr. Jour., I I, p. 89, I900. 
being inversely proportional to the sector openings. Hence it becomes necessary to determine, for each ratio of sector openings, what relative intensities will produce equal blackenings under the conditions stated.

The writer has found that the intensities are inversely proportional to the sector openings, i.e., $I / I^{\prime} \propto S^{\prime} / S$. The makers of the apparatus assume no such simple relation and propose an experimental determination of the relation for each brand of plate used. The relation is assumed to be similar to the Schwarzschild relation. The makers supply with the photometer a thin glass plate and a curve showing its $\log I / I^{\prime}$ for all frequencies. This plate was placed in front of the $180^{\circ}$ sector and a series of exposures made with the upper sector varied. On the completed negative the frequency of the points of equal blackening were read. The maker's curve supposedly gave $\log I / I^{\prime}$ and the scale reading gave $\log S^{\prime} / S$. A curve drawn with these two quantities was supposed to be the calibration curve of the sector apparatus for the brand of plate used. $\log I / I^{\prime}$ was called the "effective absorption constant" of the sector apparatus for the sector ratio $S^{\prime} / S$.

The application of the resulting curve to the writer's data on absorption gave different values for $\beta$ when the concentration or the thickness of the absorbing layer was changed. But $\beta$ can not depend on the thickness, and for the concentrations used (N/5,00o or less) Beer's law could be expected to hold. The results indicated that the absorption curve furnished with the glass plate was incorrect.

It was therefore necessary to determine the nature of the sector correction by some other method. Five nearly neutral smoke-glass plates were cut to fit the sector apparatus. The transmission of these plates, which was sensibly uniform in the yellow-green part of the spectrum, was carefully determined at $\lambda=.546 \mu$ on a spectrophotometer. Each plate was then placed in front of the sector $S$ of the photometer apparatus, and with both sectors running the setting of $S^{\prime}$ that gave the best match in the green was determined several times. The photographic plates used were sensitive for a small region on both sides of $.546 \mu$. To get greater values of $\log I / I^{\prime}$ two plates were superposed, care being taken that they were not in contact. In such a case, $\log I / I^{\prime}$ for the combination equals the sum of the separate values within a negligible error.

Fig. 2 shows the relation between $\log I / I^{\prime}$ (determined visually) and $\log S^{\prime} / S$ (determined photographically). The visual values are averages of 20 to 30 readings, while three or four determinations were made of each photographic value. The circles show $\log S^{\prime} / S$ with the lower sector running, and the crosses show $\log 360 \%$ with the lower sector at 
rest. The fact that the points lie on a straight line whose slope is unity shows that $I / I^{\prime}=S^{\prime} / S$.

As a further test of the relation, absorption photographs were made as described, using two thin glass plates over the lower sector opening, first separately and then superposed. Fig. 3 shows the curves obtained.

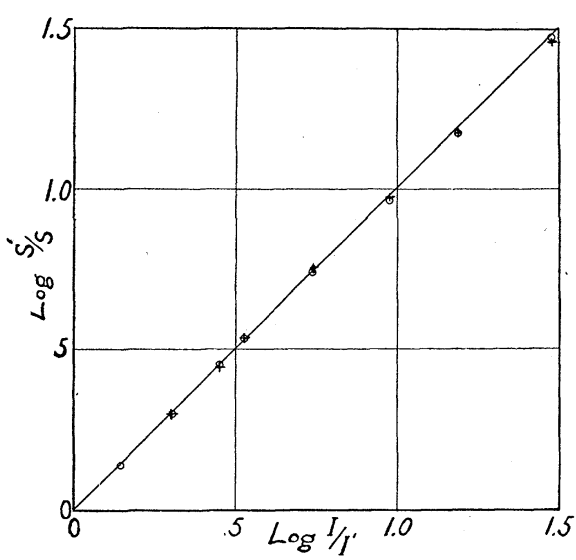

Fig. 2.

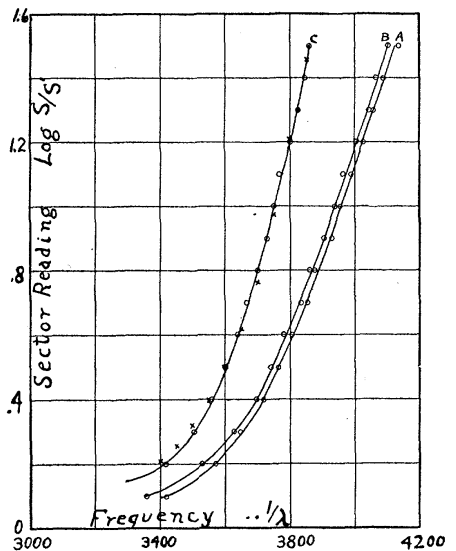

Fig. 3.

$A$, glass plate No. I; $B$, glass plate No. 2; $C$, plates I and 2 superposed.

The sum of the ordinates of the two lower curves (indicated by crosses) equals the ordinate of the upper curve for any frequency. This agreement indicates that $I / I^{\prime}=S^{\prime} / S$.

Stopping the lower sector in front of which the thin glass plate was placed and making a series of exposures with the upper sector varied, a curve was obtained with sector scale readings just .3 less than with the lower sector running. $\quad .3$ is $\log 2$. Thus, $S^{\prime} / S$ remains the same, whether $S^{\prime}$ is $180^{\circ}$ or $360^{\circ}$. The significance is that two beams can be compared by cutting down one of them with a rotating sector until a photographic match is obtained, the reading of the sector being used directly.

Fig. 5 (for fluoran) shows that different concentrations give constant $\beta$ 's for any wave-length, if we assume $I / I^{\prime}=S^{\prime} / S$. A similar assumption checks with the curve for $\beta$-orcinolphthalein (Fig. 8), which gives $\beta$ independent of thickness.

\section{EFFect of Emulsion.}

If the comparison of light intensities photographically when rotating sectors are used depends upon a relation of the Schwarzschild form, different emulsions should give different values of $\log S^{\prime} / S$ for a constant $\log I / I^{\prime}$. To investigate this possibility, the thin glass plate previously 
mentioned was used in the making of negatives with Seed's Ortho C plates two years old (two negatives), the same brand four months old (five negatives), the same with a fresh emulsion (four negatives), and a special "even sensitiveness" plate from the Eastman laboratory (two negatives). All the plates gave practically the same curve.

In only one case (Fig. 2) has it been certainly proved that $I / I^{\prime}=S^{\prime} / S$. The other experimental results might be explained on the assumption of proportionality of $I / I^{\prime}$ and $S^{\prime} / S$, and the factor of proportionality might be different for different wave-lengths. However, this factor would reasonably be expected to depend upon the sector opening and brand of plate, if upon anything. The evidence seems to the writer sufficient to justify the assumption of equality.

A similar conclusion has been reached by Weber, ${ }^{1}$ from results obtained in an entirely different manner and which seem to prove the proportionality of $I / I^{\prime}$ and $S^{\prime} / S$. In Weber's work a continuous exposure was matched against an exposure through a rotating sector. Upon cutting down the first exposure half by a geometrical screen, the sector opening that gave a match was found to be half that in the first case. This result was determined at six different wave-lengths and with several brands of plates.

The "characteristic curve" for a photographic plate, as usually plotted, gives the "density" of the plate plotted against the logarithm of the time of exposure to a source of constant intensity. Two curves which would throw light on the conclusions of this paper, and which the writer has not been able to find published, are (I) a curve in which density is plotted against the varying intensity to which the plate is exposed for equal times, and (2) a curve in which density is plotted against the openings of a sector through which exposures are made for equal times. The curves would seemingly be similar in form. It is proposed to obtain them in verification of the conclusions as to the use of a rotating sector.

The general impression of the unreliability in the use of the photographic plate for photometric work has arisen undoubtedly from the fact that in the earlier work the attempt was made to compare intensities by comparing the unequal blackenings of the plate when both the intensities and the times were different. This could not be done simply. Further confusion arose from the attempt to compare different negatives.

The above considerations as to the applicability of the photographic plate may be represented concisely in the table below, which shows three ways in which a definite amount of energy may act upon a photographic plate.

1 A. E. Weber, Ann. der Physik, 45, p. 8or, I9r4. 


\begin{tabular}{c|c|c|c|c}
\hline Intensity. & $\begin{array}{c}\text { Total Time of } \\
\text { Exposure. }\end{array}$ & Manner. & Effective Time. & Blackening. \\
\cline { 2 - 4 }$I \ldots \ldots \ldots \ldots$ & $t / n$ & Continuous & $t / n$ & $B_{1}$ \\
$I / n \ldots \ldots \ldots \ldots$ & $t$ & Continuous & $t$ & $B_{2}$ \\
$I \ldots \ldots \ldots \ldots$ & $t$ & Intermittent & $t / n$ & $B_{3}$ \\
\hline
\end{tabular}

The second case may be realized by interposing an absorbing screen that transmits only $\mathrm{I} / n$ of the light. The third case is that of a rotating sector whose angular opening is $360^{\circ} / n$. It is known that $B_{1}$ is greater than $B_{2}$ (Schwarzschild relation), and that $B_{1}$ is greater than $B_{3}$ (Abney ${ }^{1}$ ). The human eye judges the third intensity to equal the second. The results of the work discussed above show that the photographic plate integrates the third exposure in such a way as to give $B_{2}$ equal to $B_{3}$. The absorption curves are plotted on this assumption.

The Source of Light.

The arrangement of the electrical circuit for the production of a spark between aluminum electrodes under water is shown in Fig. 4. $T$ is an

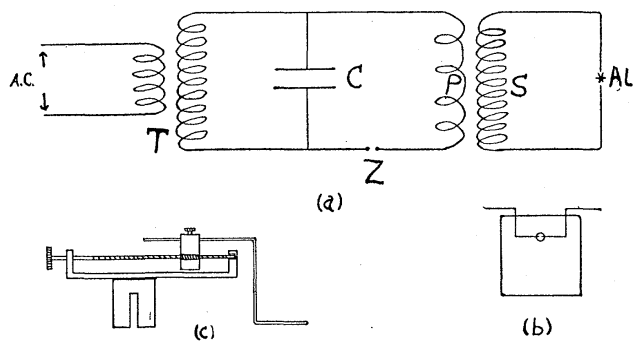

Fig. 4.

oil transformer of the closed magnetic circuit type, joined through resistance to the I Io-volt A.C. mains. The capacity $C$ consists of nine Leyden jars, each with a capacity of about $.002 \mathrm{mf}$. $P$ and $S$ represent a Tesla transformer. The primary has four turns of heavy wire forming a coil $45 \mathrm{~cm}$. in diameter, which is placed around the center of the secondary. The secondary consists of a single layer of annunciator wire wound on a wooden frame $60 \mathrm{~cm}$. long and $30 \mathrm{~cm}$. in diameter. A spark gap is at $Z$. High frequency oscillations are set up in the Tesla circuit and the discharge occurs at the gap $A l$, under distilled water.

Fig. $4(b)$ shows the arrangement of the aluminum electrodes behind a quartz window that is cemented over a hole in the side of the battery jar used to contain the distilled water, and Fig. $4(c)$ indicates the way in which the electrodes are attached and made movable. Aluminum rods $2 \mathrm{~mm}$. in diameter were used.

1 Abney, Jour. of Phot. Soc., I893-4. 
The aluminum spark is affected greatly by the action of the spark at Z. Several sorts of spark gap were tried. The best form consisted of two zinc discs cast on steel shafts and so mounted as to rotate with their edges about $3 \mathrm{~mm}$. apart. This arrangement gave a spark which was fairly uniform in action and which never failed to start when the circuit was closed. With it, a very uniform aluminum spark about $5 \mathrm{~mm}$. long could be maintained. The slight variations still evident seemed due to heating of the discs.

A small amount of tap water sufficed to render the discharge between the aluminum electrodes silent, an effect due supposedly to the increased conductivity. The collection of impurities from the air or to some chemical action produced by the spark necessitated a change of the water in the jar about once a week.

Nickel, iron, and aluminum, used as electrodes for a spark in air, all give spectra rich in ultra-violet lines, nickel being strongest in the extreme ultra-violet. Placing the electrodes under water cuts down the intensity of the extreme ultra-violet but gives a continuous background which seems to be independent of the metals used. Aluminum gives fewer lines superposed on the continuous background than either of the other metals and is therefore better. Much of the time the only lines that are easily noticeable are the two strong pairs at .396-.394 $\mu$ and $.309-.3075 \mu$. These pairs serve as landmarks when the frequency scale is laid on the negative.

The condenser circuit requires rough tuning, the intensity of the spark being increased as resonance is approached. The substitution of inductance for capacity in the Tesla primary circuit decreases the intensity without modifying the character of the spectrum. The zinc gap could be varied from $2 \mathrm{~mm}$. to $7 \mathrm{~mm}$. with a resulting increase in brightness of the source, and the aluminum spark gap could be varied from $2 \mathrm{~mm}$. to Io mm., the longer spark being most nearly free from lines. For smoothness and certainty of action the gaps were used with lengths of about $3 \mathrm{~mm}$. and $5 \mathrm{~mm}$. respectively.

The bubbles of gas formed as the water decomposes under the action of the spark are thrown violently in all directions. Often they wander back into the spark and disappear. The mechanical effect of the spark is also seen in the disintegration of the aluminum rods, which look as if battered with a hammer.

The source obtained with the above arrangement is so bright that in photographing with the Hilger instrument with the spectrograph slit $.05 \mathrm{~mm}$. wide the time of exposure for a considerable part of the spectrum need not be more than io seconds. The table below shows the relative 
times necessary to cause equal blackenings on the Seed's Ortho $\mathrm{C}$ plate used by the writer. Most of the work was done between .50 $\mu$ and $.24 \mu$, where the blackening was most uniform.

Wave-lengths.
$.50-.46 \mu$
$.46-.33$
$.33-.28$
$.28-.25$
$.25-.24$
$.24-.23$
$.23-.22$
$.22-.21$

Rela tive Exposures.
2
1
1.5
2
3
5
10
20

Discussion of the Absorption Curves.

The substances studied were chosen because they form a related group of which new compounds were being prepared in the laboratory of chemistry. Unless otherwise stated the solutions used were colorless solutions in neutral absolute alcohol. Some of the solutions become colored upon the addition of $\mathrm{KOH}$. The corresponding change in the ultra-violet absorption has been investigated in some cases and will be the subject of further study by the author.

The graphic formula for each compound is shown in connection with its curve of absorption. The benzene ring, $\mathrm{C}_{6} \mathrm{H}_{6}$, is represented by a hexagon (Fig. 5), the sides of which represent chemical bonds. At each

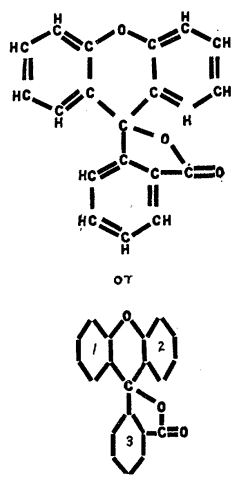

Flouran

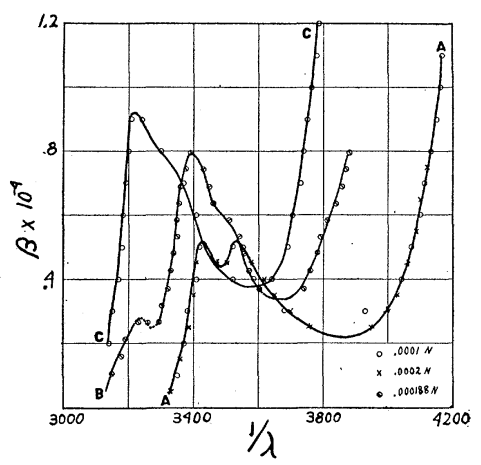

Fig. 5.

$A$, fluoran; $B$, tetrachlorofluoran; $C$, tetrabromotetrachlorofluoran.

corner of the hexagon is placed a $\mathrm{C}$ atom with its attached $\mathrm{H}$ atom. These $\mathrm{H}$ atoms may be replaced by halogen, hydroxyl, or methyl groups, etc. In the graphic formulas shown the $\mathrm{CH}$ group is not written at the corners, and where some substituted atom or group is shown attached, the connection is to the $\mathrm{C}$ atom at that point. 


\section{Flutoran.}

Fluoran, $\mathrm{C}_{2 \downarrow} \mathrm{H}_{12} \mathrm{O}_{3}$, may be considered the parent substance from which is derived a large number of colored and fluorescent compounds, such as fluorescein, eosin, etc. It contains three benzene rings (Fig. 5), and derivatives may be formed by the replacement of $\mathrm{H}$ atoms in any of these rings. Tetrachlorofluoran has four $\mathrm{Cl}$ atoms substituted for $\mathrm{H}$ atoms in the benzene ring 3 in the figure. Tetrabromotetrachlorofluoran has the further substitution of two $\mathrm{Br}$ atoms for hydrogen in each of the rings $\mathrm{I}$ and 2. The substitutions are seen to increase the absorption and shift the bands toward the visible without noticeably changing the frequency difference of the band centers.

\section{Fluorescein and its Derivatives.}

Fluorescein may be thought of as derived from fluoran by the substitution of $\mathrm{OH}$ for $\mathrm{H}$ in each of the benzene rings $\mathrm{I}$ and 2. But because the substance is colored the structure usually assigned is that shown in the formula of Fig. 6, the "quinoid" structure being assumed for related colored compounds.

Tetrachlorofluorescein has four chlorine atoms in ring 3 ; eosin (tetra.

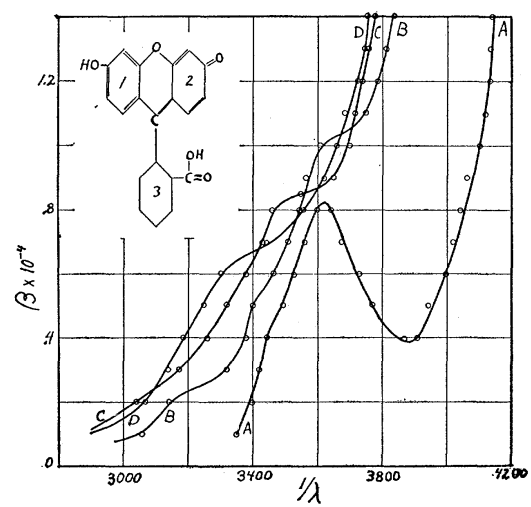

Fig. 6.

$A$, fllorescein; $B$, tetrachlorofluorescein, hydrate; $C$, eosin; $D$, tetrachloreosin.

bromofluorescein) has two $\mathrm{Br}$ atoms substituted in each of the rings $\mathrm{I}$ and 2: tetrachloreosin has both of the substitutions just mentioned. The curves show the shift toward the visible with increasing molecular weight.

The four neutral solutions were colored and all except fluorescein showed fluorescence. There is an evident difference in the form of the absorption curves for the fluorescent and non-fluorescent solutions, for which no reason can be given. Tetrachlorofluorescein was so slightly 
soluble in neutral alcohol that tetrachlorofluorescein hydrate was used.

The addition to the fluorescein solution of four molecules of $\mathrm{KOH}$ for each molecule of fluorescein produced the well-known orange color and green fluorescence, accompanied by a marked increase in the absorption in both visible and ultra-violet (Fig. 7). With the addition of the alkali,

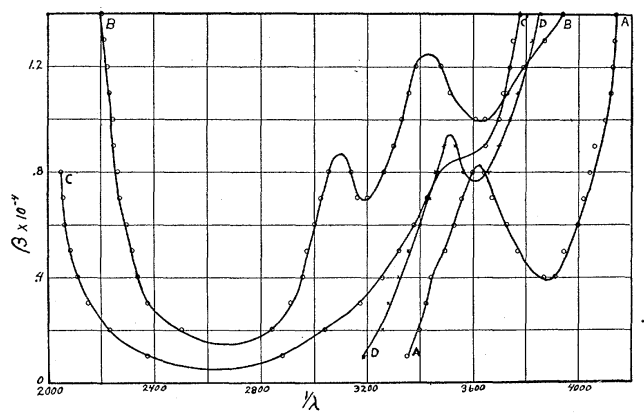

Fig. 7.

$A$, fluorescein, neutral solution; $B$, fluorescein, alkaline solution; $C$, eosin, neutral solution; $D$, eosin with trace of $\mathrm{HCl}$.

two $\mathrm{H}$ atoms in the $\mathrm{OH}$ groups are replaced by $\mathrm{K}$ atoms, forming a colored salt of fluorescein. A similar replacement of hydrogen is effected upon the addition of $\mathrm{KOH}$ to most of the colorless solutions studied, visible absorption appearing and the ultra-violet absorption being modified. The author hopes to investigate the effect of gradually increasing the amount of alkali present, to determine whether the change in absorption is a progressive shift of absorption bands or is due to the disappearance of certain bands and the appearance of new ones.

A trace of $\mathrm{HCl}$, added to the eosin solution, destroyed the yellow-green fluorescence and weakened the absorption (Fig. 7).

\section{ORCINOLPHTHALEINS.}

The orcinolphthaleins, a study of which has been published by Orndorff and Allen, ${ }^{1}$ differ in constitution from fluorescein in the substitution of methyl groups for hydrogen (Fig. 8). The $\beta$-form is represented in the formula. Isomeric $\alpha$-and $\gamma$-forms result from interchanges in the attachments of the $\mathrm{OH}$ and $\mathrm{CH}_{3}$ groups. $\beta$-orcinoltetrachlorophthalein has four $\mathrm{Cl}$ atoms substituted in the lower benzene ring and tetrabromo$\beta$-orcinolphthalein has two $\mathrm{Br}$ atoms in each of the upper rings. The neutral solutions are colorless, and their ultra-violet absorption spectra resemble that of fluorescein, though the center. of the band is shifted

${ }^{1}$ Orndorff and Allen, Jour. Amer. Chem. Soc., I7, p. I20r, I9r 5. 
toward the lower frequencies in the case of the heavier molecule. In this group also the heavier halogen causes the greater shift.

\section{HYDROQUINONEPHTHALEIN.}

This substance, though having the same percentage composition as fluorescein, differs structurally in having the two $\mathrm{OH}$ groups substituted at different places in the benzene rings. It is colorless. Its curve of

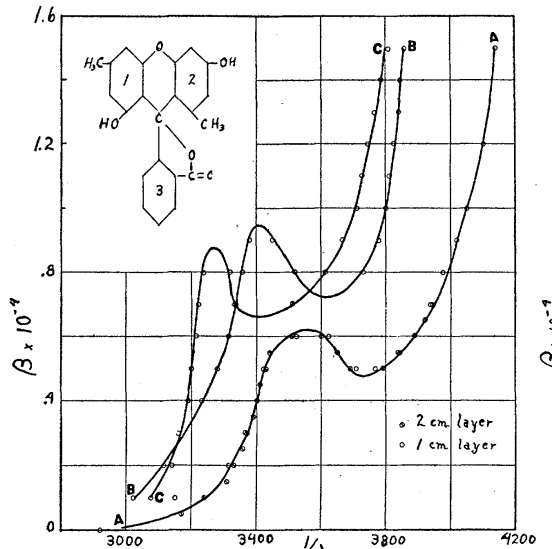

Fig. 8.

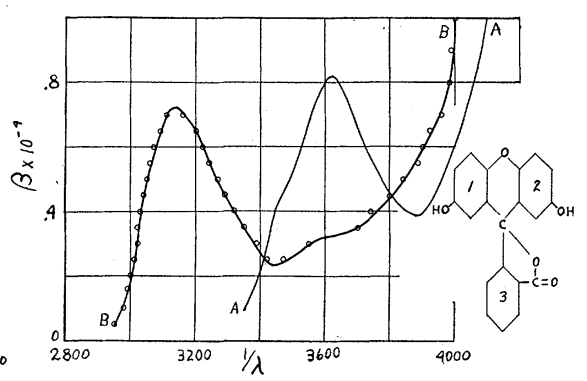

Fig. 9.

$A, \beta$-orcinolphthalein; $B, \beta$-orcinoltetrachlo- $A$, fluorescein; $B$, hydroquinonephtharophthalein; $C$, tetrabromo- $\beta$-orcinolphthalein. lein.

absorption (Fig. 9) resembles that of fluorescein in form, though the band appears at a lower frequency.

\section{Phenolphthaleins.}

Phenolphthalein la ks the bridge oxygen atom which joins the two similar benzene rings (Fig. Io). Being colorless, it is represented by a formula in which the ring 2 is not in the "quinoid" form shown in the fluorescein formula. Absorption curves for two groups of derivatives are shown. In the first group two halogen atoms are substituted in each of the upper rings. The negatives indicate a double band, with no change in the frequency difference of the band centers as the shift toward the visible takes place.

In each of the members of the second group of derivatives four $\mathrm{Cl}$ atoms are substituted in the lower benzene ring (Fig. II), the substitutions in the upper rings being as in the first group. In the phenoltetrachlorophthaleins the absorption loses its definite banded appearance, the negatives showing only shoulders on the curves. This result, appear- 

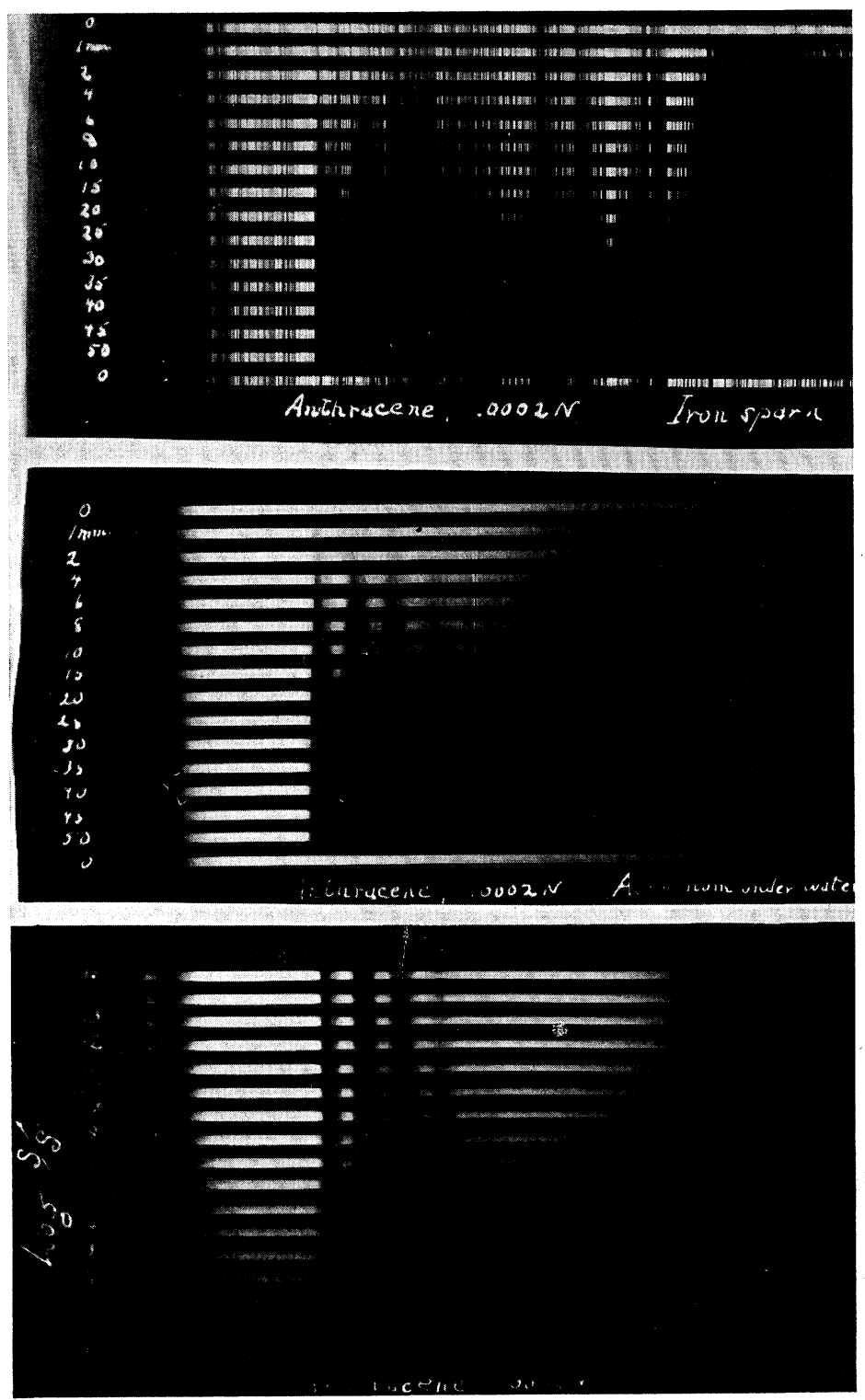

H. E. HOWE. 
ing doubtful at first, was checked up by repetition with freshly purified samples.

All of these solutions become colored by the formation of potassium salts when $\mathrm{KOH}$ is added. The effect upon the ultra-violet spectrum will be reported in another paper.

As an example of a solution that does not become colored upon the

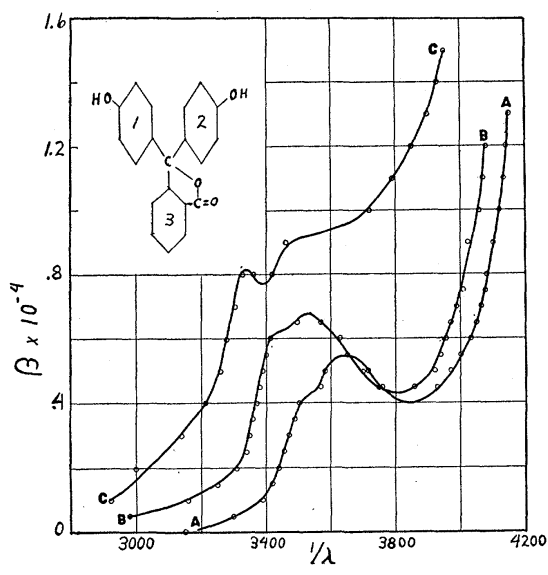

Fig. 10.

$A$, phenolphthalein; $B$, tetrabromophenolphthalein; $C$, tetraiodophenolphthalein.

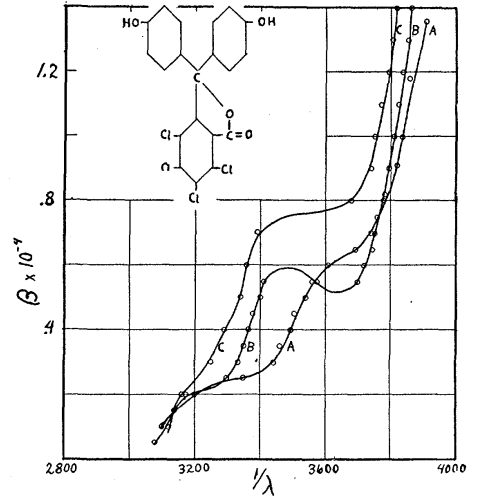

Fig. 11.

$A$, phenoltetrachlorophthalein; $B$, tetrabromophenoltetrachlorophthalein; $C$, tetraiodophenoltetrachlorophthalein.

addition of $\mathrm{KOH}$, that of fluoran may be cited. The curve for a solution containing four molecules of $\mathrm{KOH}$ to each molecule of fluoran (Fig. I2)

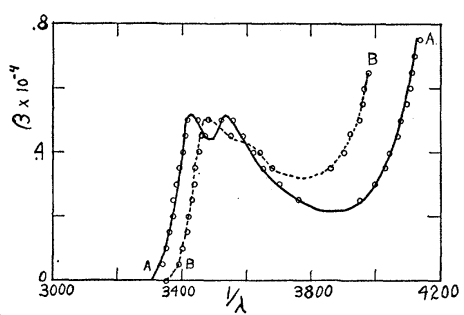

Fig. 12.

$A$, fluoran, four mol. $\mathrm{KOH} ; B$, fluoran in $N / 10$ alcoholic $\mathrm{KOH}$.

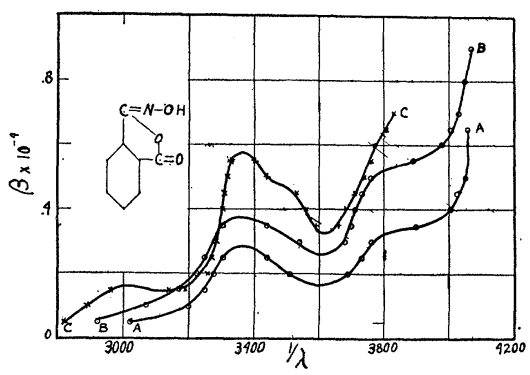

Fig. 13.

$A$, phthaloxime, white; $B$, phthaloxime, yellow; $C$, tetrachlorophthaloxime.

is not noticeably different from that of the neutral solution (Fig. 5). However, when fluoran was dissolved in a tenth normal alcoholic solution of $\mathrm{KOH}$, a modified curve was obtained. This effect was not due to the solvent, as in this case the blank cell was filled with the alcoholic solution of $\mathrm{KOH}$. 
Fig. I3 gives curves taken for comparison with curves for the same substances, as obtained by Pratt ${ }^{1}$ by the Baly-Detsch method. There is agreement as to the position of greatest absorption, but a difference as to which of the two isomeric forms absorbs most strongly. ${ }^{2}$ The third curve in the figure shows the increase of absorption upon the addition of chlorine to the molecule.

The curves given in this paper show that the molecular absorption constant is of the same order of magnitude for solutions of compounds closely related structurally. All curves were made with solutions having concentrations between .ooor $N$ and $.0002 N$. There is shown also the increasing absorption with increasing mass of the substituted groups.

In conclusion the writer wishes to thank Prof. W. R. Orndorff and Mr. S. A. Mahood for their advice concerning the study of the chemical compounds, all of which they have supplied. On the experimental side of the problem, the continued interest and frequent suggestions of Professors E. Merritt and R. C. Gibbs have been very helpful.

1 Gibbs and Pratt, Phil. Jour. Sci., 8, p. I65, I9r3.

2 Because of the difference in the coördinates used in plotting, the author was unable to show the two sets of curves on one sheet. 

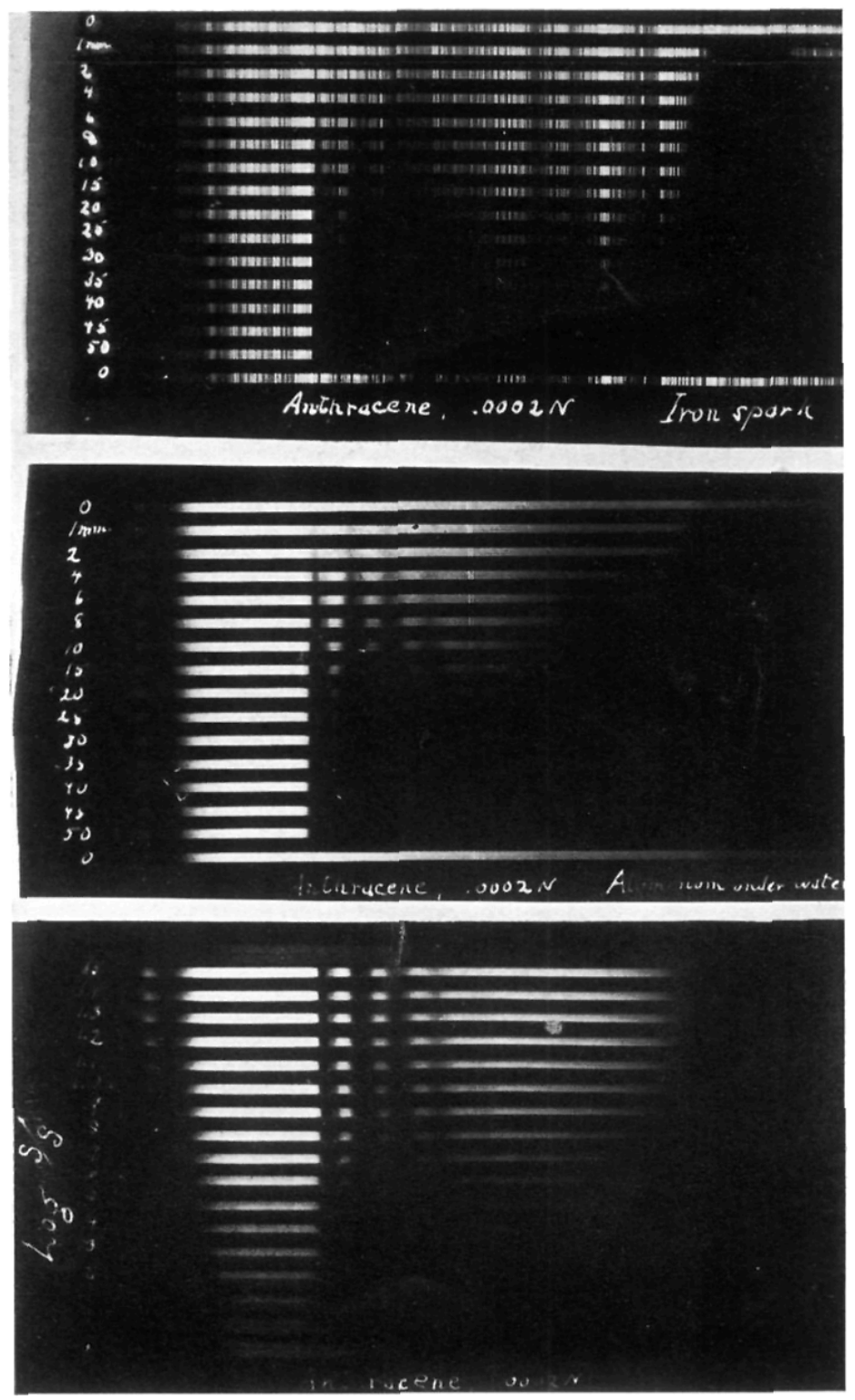